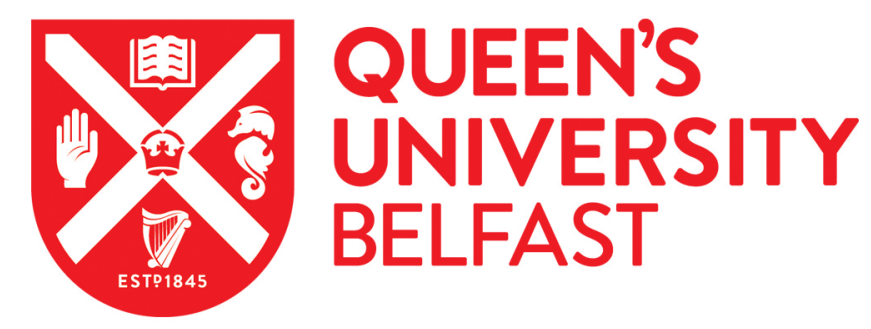

\title{
Evaluation of nonculture methods for the detection of prosthetic hip biofilms
}

McDowell, A., \& Patrick, S. (2005). Evaluation of nonculture methods for the detection of prosthetic hip biofilms. Clinical Orthopaedics and Related Research, 437(437), 74-82.

Published in:

Clinical Orthopaedics and Related Research

Queen's University Belfast - Research Portal:

Link to publication record in Queen's University Belfast Research Portal

\section{General rights}

Copyright for the publications made accessible via the Queen's University Belfast Research Portal is retained by the author(s) and / or other copyright owners and it is a condition of accessing these publications that users recognise and abide by the legal requirements associated with these rights.

Take down policy

The Research Portal is Queen's institutional repository that provides access to Queen's research output. Every effort has been made to ensure that content in the Research Portal does not infringe any person's rights, or applicable UK laws. If you discover content in the Research Portal that you believe breaches copyright or violates any law, please contact openaccess@qub.ac.uk. 


\title{
Evaluation of Nonculture Methods for the Detection of Prosthetic Hip Biofilms
}

\author{
Andrew McDowell, PhD; and Sheila Patrick, PhD
}

\begin{abstract}
Authors of previous studies have found that culture-based detection of prosthetic hip infections can be improved by adherence to strict anaerobic protocols and mild ultrasonication of retrieved prostheses to remove bacteria growing as an adherent biofilm. Furthermore, direct analyses of sonicate samples by immunofluorescence microscopy (with genus-specific and species-specific monoclonal antibodies or polyclonal antisera) produces significantly greater and more rapid detection rates for infection compared with culture alone. Despite its diagnostic value within a research setting, the practical advantages and limitations of immunofluorescence microscopy for a busy diagnostic laboratory needs consideration. In addition, the method must be evaluated against other nonculture-based techniques that may prove more appropriate. In this review, we describe how immunofluorescence microscopy presents an attractive and reliable method for routine detection of prosthetic hip biofilms compared, primarily, to polymerase chain reaction, although other nonculture methods of diagnosis based on fluorescent in situ hybridization and serologic analysis are considered. We describe how the immunofluorescence microscopy technique is robust, relatively simple and, in contrast to broad-range $16 \mathrm{~S}$ recombinant-deoxyribonucleic-acid-based polymerase chain reaction, does not require a separate clean-room facility or strict adherence to aseptic techniques. More importantly we highlight how dislodged biofilm, which appears as large aggregates of bacteria, easily can be identified from skin contaminants that occur as single cells or small aggregates of only a few cells.
\end{abstract}

Total hip replacement surgery is a medical success story and has improved the quality of life considerably for countless people. The majority of patients who receive a prosthetic hip implant have dramatic and immediate relief

From the Department of Microbiology and Immunobiology, School of Medicine, Queen's University, Belfast, Northern Ireland.

Both authors received funding from the Northern Ireland Health and Personal Social Services Research and Development Office.

Correspondence to: Sheila Patrick, PhD, Department of Microbiology and Immunobiology, School of Medicine, Queen's University, Grosvenor Road, Belfast, BT12 6BN, Northern Ireland. Phone: 44(0)2890 632512; Fax: 44(0)2890 635024; E-mail: s.patrick@qub.ac.uk.

DOI: 10.1097/01.blo.0000175123.58428.93 of pain and regain satisfactory hip function and quality of life. ${ }^{5}$ However, a proportion of patients do develop various complications that require further surgery and replacement of the prosthesis (revision arthroplasty). ${ }^{27}$ The morbidity and trauma associated with revision arthroplasty is considerable, and in the United Kingdom, incurs large costs to the National Health Service. Although aseptic biomechanical loosening is thought to be the most common underlying cause for prosthetic hip replacement, revision arthroplasties caused by prosthetic hip-associated infections also constitute a substantial burden to the healthcare system and are a disastrous complication for the surgeon. ${ }^{13} \mathrm{Un}$ fortunately, the rate of infection-related failure for second implants is thought to be even higher than that seen with primary revision surgery. ${ }^{7}$ This may be a consequence of the longer operating times involved or unrecognized infection at the time of the revision surgery, which leads to implantation of the second prosthesis without debridement and antimicrobial intervention. ${ }^{36}$ In contrast, the incorrect suspicion that a prosthetic hip implant may be infected at the time of revision surgery not only increases the anxiety and stress to the patient, but also results in additional surgery, a prolonged hospital stay, and extra expense. As a consequence, early and accurate diagnosis of prosthetic hip infections would serve to improve the treatment and management of patients considerably.

Although different methods have been used to detect the presence of prosthetic hip infections, no standardized protocol or set of diagnostic guidelines has been universally adopted. Most patients routinely are assessed for the presence of infection before revision surgery using a polyphasic approach that includes clinical history and examination of the patient, measurement of leukocyte count, erythrocyte sedimentation rate and C-reactive protein levels, radiographic and radionuclide imaging, and culture analysis of synovial fluid aspirated from the hip joint (aspiration arthrography). ${ }^{15,24,36,39}$ Postoperatively, detection of infection normally is investigated by routine culture analysis of the explanted prosthesis and periprosthetic tissue samples. ${ }^{36}$ Authors of histopathology studies also have provided evidence that the presence of inflammatory cells 
in periprosthetic tissue biopsies is a valuable marker for the presence of infection. Indeed, researchers who have participated in the Oxford Skeletal Infection Research and Intervention Service (OSIRIS) in the United Kingdom have recommended that patients having revision surgery routinely are evaluated for infection by histologic screening. ${ }^{2,26}$ Although the combined use of all these methods usually is sufficient to detect the presence of acute infection that may occur soon after surgery, their diagnostic sensitivity for the detection of chronic low-grade infection may be problematic.

Against this background, we now review nonculturebased methods for the routine detection and identification of prosthetic hip infections. We initially describe how strict anaerobic processing of retrieved prostheses followed by the detection of dislodged prosthetic hipassociated biofilms can improve the diagnosis of prosthetic hip infections. We then highlight the value of nonculture-based methods for the detection of such infections and compare polymerase chain reaction (PCR)-based methods and fluorescent in situ hybridization (FISH) and serologic analysis with immunofluorescence microscopy (IFM). We conclude that IFM currently provides the best diagnostic platform for the detection of prosthetic hip infections and describe how the technique allows dislodged biofilm, which appears as large aggregates of bacteria, to be differentiated from skin contaminants that occur as single cells or small aggregates of only a few cells.

\section{Detection of Prosthetic Hip Joint Biofilms}

Authors of previous studies have provided evidence that the incidence of prosthetic hip joint infection is grossly underestimated by various diagnostic methods routinely practiced in clinical laboratories. ${ }^{37,38}$ In these studies, infection was identified as a major cause of prosthetic joint failure in 26 of $120(22 \%)$ retrieved prostheses examined. ${ }^{37,38}$ Organisms cultured from the prostheses primarily were coagulase-negative staphylococci, mainly Staphylococcus epidermidis and Propionibacterium acnes, which are associated with chronic low-grade infections. When the patient's notes from 18 of these 26 individuals were scrutinized, it was apparent that infection only had been suspected in six cases (33\%). Furthermore, in only two cases were bacteria cultured by the routine diagnostic laboratory from preoperative aspirates or periprosthetic tissue removed at the time of surgery. This considerably increased culture-based detection of infection was achieved by immediate placement of the retrieved hip prosthesis in an anaerobic atmosphere, mild ultrasonication $(5 \mathrm{~min}, 50 \mathrm{kHz}$ ) of the prosthesis in prereduced quarter-strength Ringer's solution, containing cysteine, to dislodge bacteria growing on the surface as an adherent biofilm, and strict anaerobic processing of the resulting sonicate samples. In addition, direct sampling of sonicate samples by IFM, with a monoclonal antibody (MAb) specific for $\mathrm{P}$. acnes and a polyclonal antiserum specific for Staphylococcus spp., revealed the presence of bacteria in $63 \%$ of revised prostheses, providing evidence that culture-based analyses alone may not be the most sensitive diagnostic technique. Molecular detection of infection by broad range $16 \mathrm{~S}$ recombinant deoxyribonucleic acid (rDNA)-based PCR, using universal primers, seemed to confirm this view because positive PCR results were obtained for $72 \%$ of the revised prosthetic hip samples. Although neutrophilic infiltration in periprosthetic tissues has been recommended as a useful nonculture method for the detection of prosthetic hip joint infections, eight of the culture-positive cases (30\%) identified in these studies were found to be negative for the presence of neutrophils in prosthetic-hip-associated tissue samples (where available). ${ }^{37,38}$ In contrast, lymphocytes and/or macrophages were present, which could be explained, in theory, by the slow release of bacterial components from the prosthetichip-associated biofilm. Histopathologic examination of tissue taken from culture-negative patients revealed the presence of high-level neutrophilic infiltration (10 or more neutrophils per high-power field) in eight samples. A further 36 samples with low levels of neutrophils or none at all had high numbers of lymphocytes or macrophages (10 or more lymphocytes or macrophages per high-power field). For a number of these samples, although the tissue pathology score was high, bacteria were present in small numbers by IFM. It therefore remains to be established whether the observed inflammatory response in such samples was driven by wear debris from the prosthesis, ${ }^{34}$ bacterial components, or indeed a combination of both. On the basis of these data it has been recommended that current methods routinely used for the detection of prosthetic hip infections be reassessed. ${ }^{37,38}$

\section{Importance of Nonculture Methods for Detection of Prosthetic Hip Joint Biofilms}

Because of shorter processing times, nonculture-based methods, such as broad-range 16S rDNA-based PCR and IFM, provide the opportunity for earlier postsurgical detection and identification of prosthetic hip biofilms compared with conventional culture-based approaches, especially those involving anaerobic growth (Fig 1). More importantly, such techniques can facilitate the detection of viable but nonculturable infections in prosthetic hip joints and periprosthetic tissue and bone samples. This may occur because of low numbers of organisms, the use of prophylactic antibiotic therapy, or the fact that bacteria growing within a biofilm community can enter a state of dormancy or quiescence caused by inappropriate growth conditions, which contributes to their increased resistance 


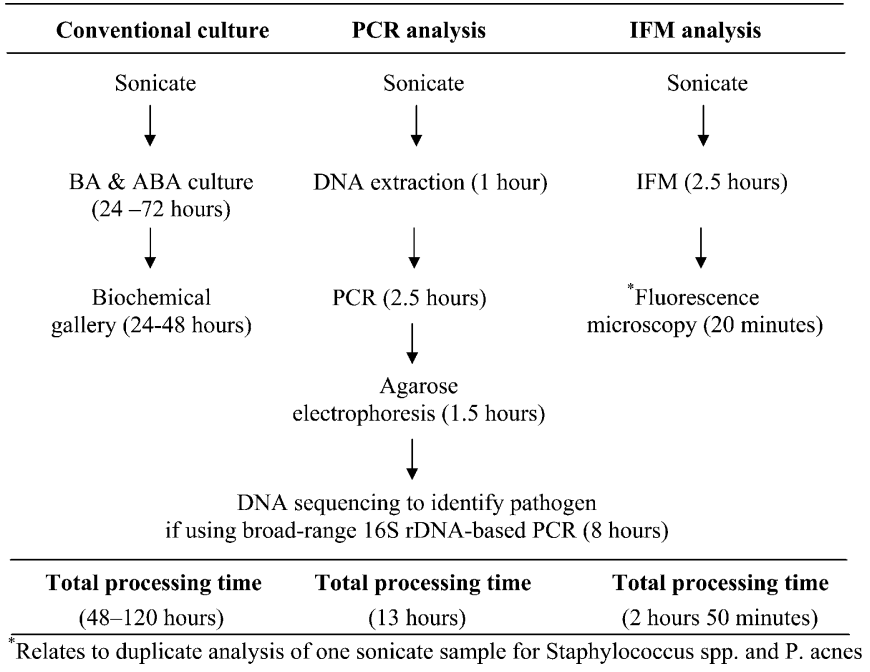

Fig 1. A comparison of conventional culture, PCR, and IFM processing times for the detection and identification of prosthetic hip infections in the routine diagnostic laboratory is shown. Sonicate samples are prepared by mild ultrasonication ( $5 \mathrm{~min}, 50 \mathrm{kHz}$ ) of the retrieved prosthesis in prereduced 1/4strength Ringer's solution containing cysteine.

to antimicrobials. ${ }^{11,31}$ Also, it cannot be discounted that organisms growing as a biofilm may become so highly adapted to their environment that the fastidious growth requirements necessary for their cultivation are not provided within the laboratory setting. ${ }^{37}$ Clearly, the contribution that nonculture-based methods make to the detection of prosthetic hip joint infections should not be undervalued. Their capacity to provide faster post-surgical detection of infection, as well as the identification of patients with prosthetic hip infections that traditional approaches may have missed, will facilitate earlier and more aggressive antimicrobial intervention and therefore will educe the possibility that a new infection will be established. This will substantially contribute to the universal goal of improving and refining patient treatment and care.

\section{Comparison of Nonculture-Based Methods for the Detection of Prosthesis Hip Joint Biofilms}

\section{Polymerase-Chain-Reaction-Based Protocols}

Although nonculture-based methods may prove to be valuable for the detection of prosthetic hip joint biofilms within a research setting, the practical advantages and limitations of such approaches for routine detection of such infections within the context of a busy clinical microbiology laboratory must be considered. Because most modern diagnostic laboratories now have access to thermal cyclers it is only natural that nonculture detection methods based on PCR technology are adopted. Such an approach is even more obvious when one considers that universal or broad-range 16S rDNA-based PCR, using highly conserved oligonucleotide primers, provides an ideal molecular platform for the detection of almost all types of bacterial infection with normally high sensitivity. ${ }^{10,21,43}$ However, despite the initial attractiveness that PCR may offer for the detection of prosthetic hip joint infections, a number of potential caveats associated with the use of the technique, especially within a routine laboratory, do exist. Most notably, the exquisite sensitivity that is often associated with PCR detection methods can be a considerable burden practically and economically for the diagnostic laboratory, which must ensure that the positive result obtained for a patient's sample is not caused by coamplification of contaminating DNA. ${ }^{4,22}$ Indeed, based only on this, many of laboratories have decided not to adopt broadrange 16S rDNA-based PCR as part of their diagnostic service. Potential DNA contamination can arise from a variety of different sources, including nonsterile sites surrounding the clinical specimen, the specimen vial, surgical or laboratory personnel, aerosols and reagents used for the extraction of DNA and PCR setup, and even unrelated PCR activities in adjacent laboratories. ${ }^{4,22,32,43}$ In addition, contamination from pipettes and other equipment, such as PCR cabinets and reaction vials, also can occur. The detection of contamination from nonsterile sites particularly is an important consideration when analyzing retrieved prosthetic hip joints because the organisms frequently responsible for infection, such as Staphylococcus spp, also are part of the normal skin microbiota. To help avoid contamination during broad-range 16S rDNA-based PCR, a wide range of preventative measures need to be taken. These include the use of molecular grade reagents that must be screened for artifactual DNA before use, the wearing of sterile gloves and protective clothing, and dedicated rooms for DNA extraction and PCR setup that contain safety cabinets fitted with ultraviolet lamps (for DNA degradation), pipettes, filtered pipette tips, reaction vials, and other general equipment that must remain on site. ${ }^{4,22}$ Elimination of potential contaminants from PCR reagents and equipment can also be achieved by enzymatic and chemical treatments. Furthermore, when carrying out experiments it is imperative that appropriate positive and negative controls (for DNA extraction and PCR setup) are included in each diagnostic run. The use of internal amplification controls also will serve to highlight any falsenegative results that may arise from PCR inhibitors within the clinical sample. ${ }^{43}$ Although the use of control samples provides some reassurance that contamination of clinical samples has not occurred, it does not completely eliminate such a possibility. For example, we recently identified the presence of artifactual DNA from a $\beta$-proteobacterium in batches of sterile irrigation fluid routinely used during 
prosthetic hip surgery. Retrospective sequencing studies now also have identified the presence of this DNA in a number of sonicate samples analyzed in our earlier study. ${ }^{37}$ As a consequence, the $72 \%$ detection rate previously reported for the presence of bacteria in failed prosthetic hip implants by broad-range 16S rDNA-based PCR was an overestimate. The detection of a number of falsepositive samples, even when all controls gave expected results, strikingly highlights the potential complications that can arise when using a broad-range 16S rDNA-based PCR approach.

Researchers who conducted previous studies in our laboratory showed that PCR can be used reliably to detect Staphylococcus organisms to concentrations of $10^{4}$ $\mathrm{cfu} / \mathrm{mL}$, whereas detection limits for P. acnes reliably do not go below $10^{5} \mathrm{cfu} / \mathrm{mL}^{37}$ To date, results of ongoing studies with various DNA extraction protocols and broadrange $16 \mathrm{~S}$ rDNA primer sets have not improved on these detection limits (data not published). Detection limits for the PCR-based diagnosis of gram-positive bacteria can be problematic because of the robust nature of the cell envelope, which is composed of a 3-dimensional matrix of polymers, primarily peptidoglycan and techoic and/or lipoteichoic acids. In addition, P. acnes is a member of the actinomycetales, which are known to have additional cell envelope polymers, making them even more robust than other Gram-positive bacteria. This may therefore explain, at least in part, why it is especially difficult to optimally extract P. acnes genomic DNA. Although this relatively poor detection limit may negate the possibility that low levels of P. acnes contamination from nonsterile sites or surgical or laboratory personnel would be detected, it also does raise the possibility that smaller numbers of the organism that may be present in a chronic infection would be missed. Although the use of a nested or semi-nested PCR method undoubtedly would improve the detection of $\mathrm{P}$. acnes, we are uncomfortable with such an approach for diagnosis because of the greater risk of cross-contamination (from first-round amplification) during routine processing.

Despite the fact that broad-range 16S rDNA-based PCR offers the potential to detect all types of bacterial organisms it does not directly identify the specific nature of the infection without further laboratory investigations. This usually is achieved by DNA sequence analysis of the $16 \mathrm{~S}$ rDNA amplicon followed by comparison of the sequence to those previously deposited in global sequence databases, such as GenBank at the National Center for Biotechnology Information, and the Ribosomal Database Project. ${ }^{6,14}$ However, in addition to increasing the laboratory processing time (Fig 1), this approach will not work when a polymicrobial hip infection is present. In such circumstances, PCR amplicons for each species present in the sample may be generated leading to nonsensical DNA sequences that cannot be interpreted. To overcome this problem, amplicons would have to be separated, either by cloning or some form of polyacrylamide gel electrophoresis, which would delay identification and further increase processing times. ${ }^{41,42}$

Alternate PCR approaches are available to the molecular microbiologist to circumvent the problems associated with the broad-range 16S rDNA-based approach for detection of prosthetic hip biofilm. These include the use of genus or species-specific primers, which serve to reduce, although not eliminate, the potential problems that could arise from contamination. The use of species-specific primers would also negate the necessity for subsequent DNA sequence analysis as the organism could be directly identified from the positive PCR result. Such specific primer sets still can be based on the 16S rDNA locus, although other nonribosomal targets frequently are used with great success. ${ }^{12,18}$ These alternate targets are often less well conserved than $16 \mathrm{~S}$ rDNA sequences affording more opportunity to design specific primers that will differentiate closely related organisms. For example, we recently found that P. acnes serotypes I and II were almost indistinguishable based on 16S rDNA sequences. ${ }^{20}$ However, analysis based on the recA housekeeping gene revealed considerably greater serotype-specific polymorphisms that will facilitate the generation of diagnostic primers specific for each type. ${ }^{20}$ Another example is the glyceradehyde-3-phosphate dehydrogenase gene, which is a useful tool for the differentiation of most Staphylococcus spp. ${ }^{45}$ One obvious disadvantage with the use of genusspecific or species-specific primers would be the possibility that certain pathogens may not be detected from an infected hip prosthesis. To reduce this possibility, multiple primer sets specific for the organisms most likely to be detected would have to be used. Realistically, this would include primers for various Staphylococcus spp., primarily $\mathrm{S}$. aureus and S. epidermidis, and primers for P. acnes. The use of genus-specific primers for Staphylococcus spp. also may be useful and would highlight the presence of other species in addition to $\mathrm{S}$. aureus and $\mathrm{S}$. epidermidis. These individual PCR tests could be combined into a multiplex assay to save time during setup and also reduce the number of samples to process. ${ }^{12,19}$ However, optimization of such assays can prove problematic depending on the number of primer sets to be used and their compatibility with respect to annealing temperatures. Also, each primer set must generate different product sizes that are easily distinguished on an agarose gel.

\section{Fluorescent In Situ Hybridization}

In addition to PCR, the discriminatory potential of $16 \mathrm{~S}$ rRNA sequences also could be used for the detection of 
prosthetic-hip-associated bacteria by FISH. In this technique, a single oligonucleotide probe (15-30 nucleotides) that has been linked covalently to a fluorescent dye at the $5^{\prime}$ end is hybridized to ribosomes present within the bacterial cell. ${ }^{1,23,30,40}$ After stringent washing, the stained cells are detected by fluorescence microscopy or flow cytometry. Fluorescent in situ hybridization not only provides the opportunity to observe and identify microbial cells within clinical samples, but, in combination with confocal laser scanning microscopy, it can be used to reconstruct the spatial arrangement of infecting organisms within their habitat. ${ }^{40}$ As with broad-range 16S rDNAbased PCR, universal probes that will react with all bacteria can be used or, alternatively, more specific probes can be designed that discriminate between different bacterial species. ${ }^{30}$ The method has been used to study complex microbial communities in the oral cavity, the gastrointestinal tract, and infections of the respiratory tract and pathogenic organisms in blood culture bottles. ${ }^{23}$ Although this technique is appealing for the detection of prosthetic hip biofilms because of its potential for direct observation of bacteria, and the relative low cost and ease with which different oligonucleotide probes can be produced, there are a number of practical problems that remain to be overcome. In particular, autofluorescence of the organisms themselves, or material surrounding the bacteria, can decrease the signal-to-noise ratio and hinder detection of specific fluorescent signals. ${ }^{23}$ Also, the complex nature of the cell envelope in gram-positive bacteria, especially those exhibiting long or medium chain mycolic acids, and the large extracellular polysaccharide capsules present on some gram-negative organisms, can create problems relating to insufficient penetration of the oligonucleotide probe into the bacterial cell. ${ }^{23,30,40}$ The recent introduction of peptide nucleic acid probes, which have an uncharged polyamide backbone, may help to reduce this problem because they have the capacity to diffuse through hydrophobic cell walls. ${ }^{25,28}$ However, the expensive nature of such probes and problems associated with their specificity currently are hindering their widespread application. Problems also can arise because of the accessibility of the oligonucleotide probe to target sites on the 16S rRNA molecule. This occurs because of loop and hairpin formations in the higher-order structure of the ribosome and protein-rRNA interactions. The use of unlabelled helper probes, which bind adjacent to the probe target site and are thought to open up higher order structures that hinder binding, can enhance detection. ${ }^{9}$ Other issues that can arise when using FISH include the relationship between ribosome number and fluorescent signal. The content of rRNA can vary considerably between cells of a given strain because of their physiologic state, which is dependent on their phase of growth. ${ }^{30}$ This particularly is per- tinent to slow-growing or dormant biofilm bacteria in which ribosome numbers likely are to be low. As a consequence, low-signal intensity or a false-negative result may occur. Attempts to address this issue have focused on increasing cellular ribosome content by incubating samples with a cocktail of substrate and antibiotics that cause cell activation and rRNA synthesis without cell division. ${ }^{16}$ Although this can lead to an increase in signal intensity, the caveat with such an approach is the selectivity of the substrates and antibiotics. Consequently, although FISH remains an extremely useful research tool, its application to routine diagnosis in the short term may be troublesome.

\section{Serologic-Based Analysis}

Nonculture-based serological detection of gram-positive infections on prosthetic hip implants by enzyme-linked immunosorbent assay (ELISA) also has been described. ${ }^{29}$ This method measures the serum immunoglobulin $\mathrm{G}$ response to a short-chain length form of lipoteichoic acid that is present in the cell membrane and extracellular material of Staphylococcus spp. The technique also has been applied to the detection of gram-positive infections associated with intravenous catheters and sciatica. ${ }^{8,33}$ The sensitivity (93.3\%) and specificity (96.9\%) of the ELISA has provided evidence that it could be useful in the detection of occult prosthetic hip infections. Furthermore, because the method is quick and relatively inexpensive, it may be easily adapted into the diagnostic laboratory. However, authors of the original ELISA study only examined 15 patients with proven prosthetic hip infections; therefore evaluation of this technique with a much greater number of patients would be required to fully assess its diagnostic value. ${ }^{29}$ Furthermore, as the technique is not specific for prosthetic hip infections; patients with other bacterial infections at the time of analysis or those with a recent history of infection may not be suitable for testing. A further problem with this method is the presence of antibodies to the staphylococcal antigen within the normal population. As a consequence, basal $\operatorname{IgG}$ titer levels need to be evaluated for the population being studied. The determination of a suitable titer cut-off point is somewhat subjective, and the possibility also remains that titers may overlap between the patient and control groups.

\section{Immunofluorescence Microscopy}

An alternative nonculture detection method for prosthetic hip biofilms based on IFM is being developed (Fig 2). ${ }^{37} \mathrm{In}$ this approach, adherent biofilms dislodged from the prosthesis by mild ultrasonication are concentrated and directly fixed to the wells of a glass slide before incubation with a $\mathrm{MAb}$ or polyclonal antisera specific for the organism(s) 


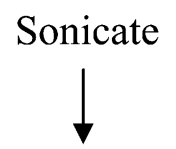

Fixing step $\left(10 \mathrm{~min}\right.$ at $\left.-20^{\circ} \mathrm{C}\right)$

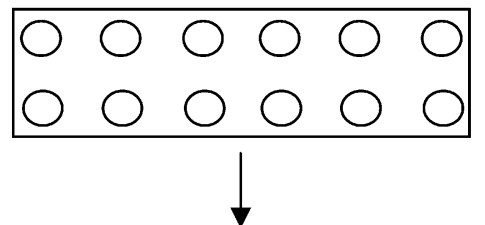

$\mathrm{MAb}$ / Polyclonal antisera $\left(45 \mathrm{~min}\right.$ at $\left.37^{\circ} \mathrm{C}\right)$

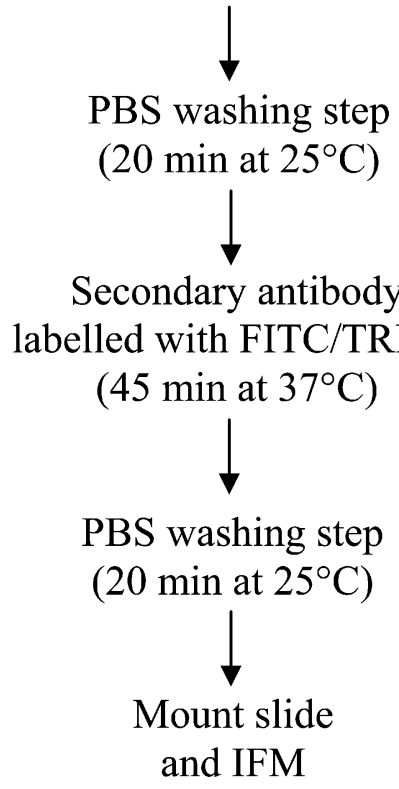

Fig 2. An IFM-based protocol for the detection and identification of prosthetic hip infections is shown. Sonicate samples are prepared by mild ultrasonication ( $5 \mathrm{~min}, 50 \mathrm{kHz}$ ) of the retrieved prosthesis in prereduced $1 / 4$-strength Ringer's solution containing cysteine.

under investigation. After incubation with a secondary antibody conjugated to a fluorogenic dye, such as fluorescein isothiocyanate (FITC) or tetramethylrhodamine isothiocyanate (TRITC), bacteria present in the samples can be detected by fluorescence microscopy (indirect labeling). With specific regard to the detection of prosthetic hip biofilms, we think this approach has a number of key advantages over a PCR-based method and, consequently, may be more suitable for routine diagnostic laboratories. In particular, the current IFM method has a considerably shorter processing time compared with PCR, which means the clinician can be informed of any underlying infection within an earlier timeframe (Fig 1). ${ }^{37}$ Also, the IFM-based method provides the opportunity to distinguish bacterial contamination from a real infection, which is one of the critical advantages of the technique. In conventional PCR, the basis of a positive result is the detection of a band of the correct size on an agarose gel. However, even when all positive and negative control samples are correct, there still remains the possibility that the clinical specimen itself could have been contaminated, which observation of a DNA band will not highlight. In contrast, with IFM the bacterial cells can be seen directly (with coryneform or cocci morphology), which facilitates identification of contaminating organisms from those that have been growing as an adherent biofilm on the prosthesis. On the basis of control experiments conducted in our laboratory, as well as our experience processing large numbers of retrieved hip prostheses, organisms that we normally would classify as contaminants are observed infrequently within the well of the slide and occur either as single cells or a small aggregates composed of only a few cells. ${ }^{37}$ In contrast, dislodged biofilm can be easily identified as large aggregates of bacterial cells consisting of several layers (Fig 3). These bacterial aggregates, which vary in depth from 3.5 to $4.0 \mu \mathrm{m}$, are only ever seen after sonication, but never after a simple diluent wash of the prosthesis. ${ }^{37}$ Because of the differences in bacterial morphology for coryneform and cocci species, the combined use of antibodies specific for P. acnes and Staphylococcus spp. also enables any dislodged biofilm containing both types of organisms to be observed (Fig 4). Although the use of specific MAbs or polyclonal antisera enables the nature of the infecting organism(s) to be directly identified, it does create the possibility that other organisms unreactive with the antibodies would not be identified. Although this is a potential limitation of the technique, the introduction of additional MAbs or antisera raised to other putative pathogens would improve this situation. To illustrate this point, we have improved our detection of prosthetic hip infections by generating MAbs specific for the two serotypes of P. acnes (Types I and II). ${ }^{20}$

We have found IFM-based detection of prosthetic hip biofilms to be a very robust technique that does not require much expertise or training to do. Apart from the initial cost of a fluorescence microscope, the method is relatively inexpensive and does not require the scrupulous adherence to aseptic technique that is required to eliminate DNA contamination when using broad-range 16S rDNA-based PCR. Indeed, the technique can be conducted comfortably on the laboratory bench once the sonicate sample has been prepared and fixed to the microscope slide. Furthermore, our IFM method does not suffer from the various problems that can arise when using a FISH-based approach, such as insufficient penetration of the oligonucleotide probe into the bacterial cell or accessibility of the oligonucleotide probe to target sites on the $16 \mathrm{~S}$ rRNA molecule. As a 

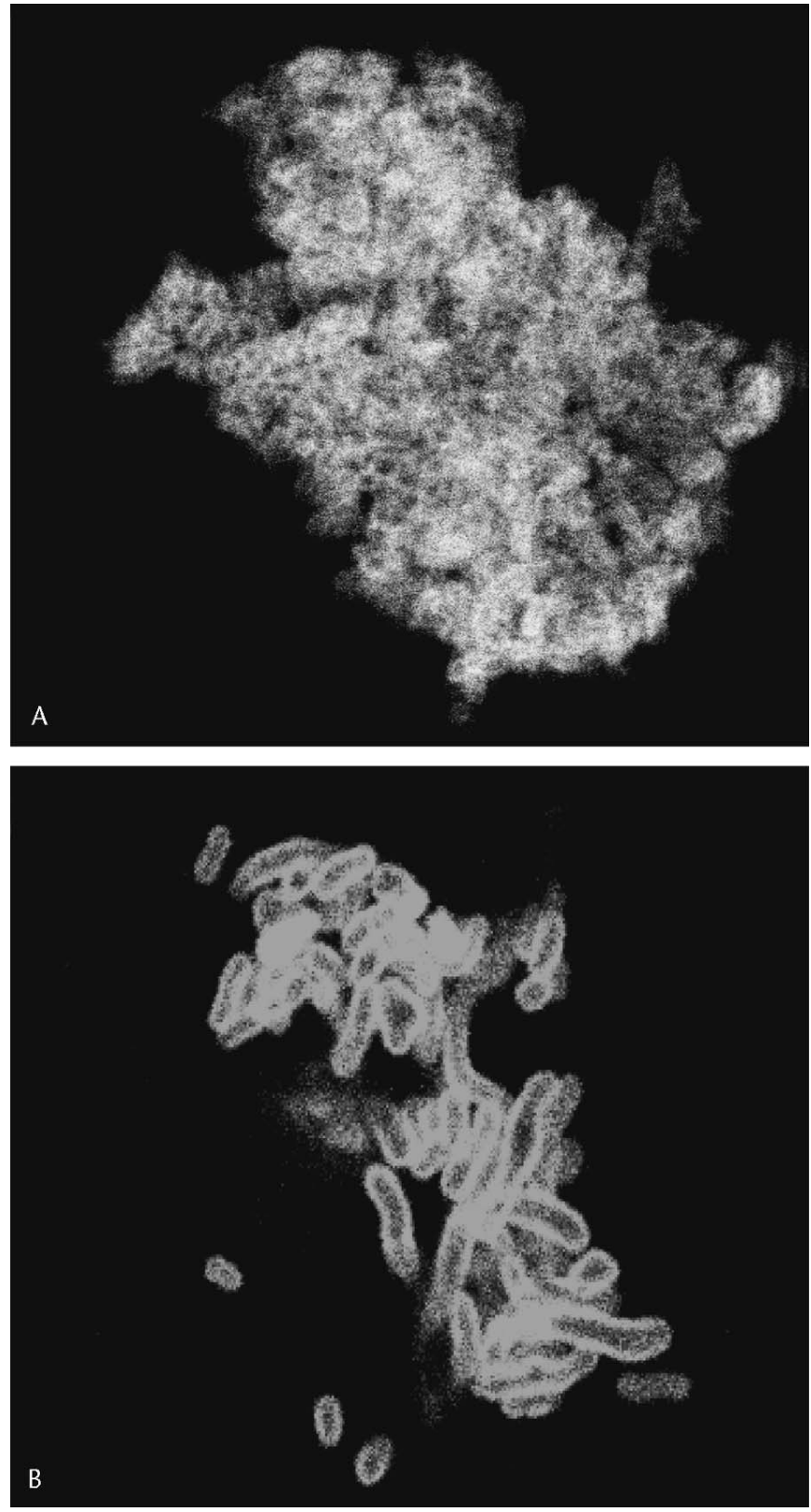

Fig 3. A confocal laser-scanning micrograph of bacterial biofilms removed from retrieved prostheses by ultrasonication is shown. Biofilms were labeled with either (A) anti-Staphylococcus spp. antiserum (1/400) or (B) undiluted anti-P. acnes MAb hybridoma culture supernatant (QUBPa3) before incubation with a secondary antibody conjugated to a fluorescent dye (FITC).

consequence, the method represents an attractive nonculture-based method that could be implemented, at least in the short term, for the detection of prosthetic-hipassociated infections. The IFM technique also could be applied to other medical devices used in orthopedics, such

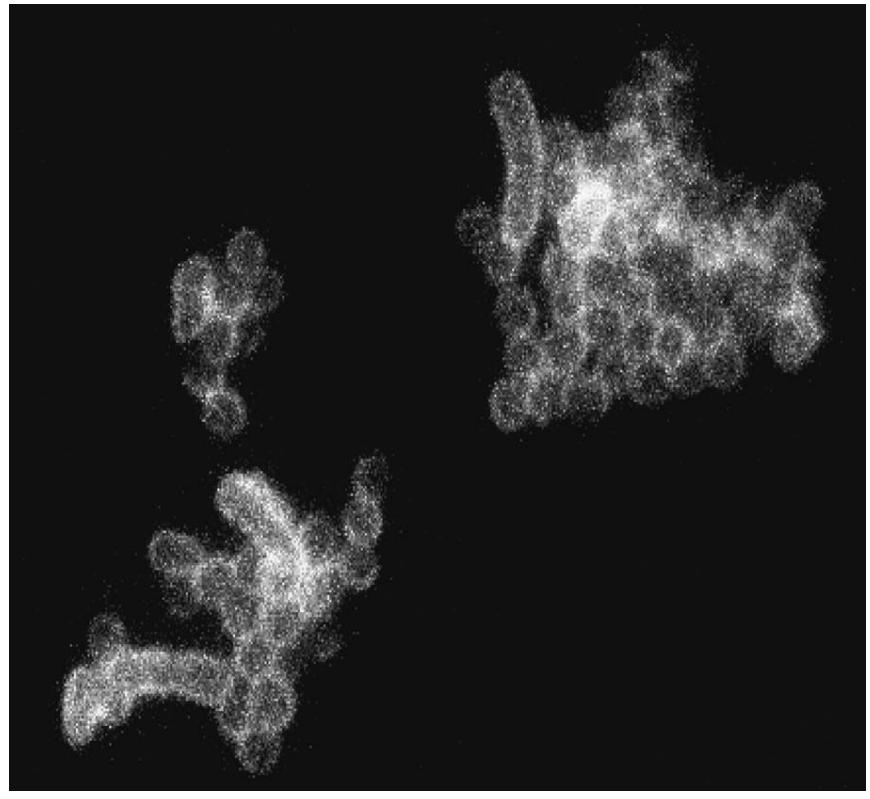

Fig 4. A confocal laser-scanning micrograph of a bacterial biofilm dislodged from a retrieved prosthesis by ultrasonication is shown. The biofilm was labeled with an anti-Staphylococcus spp. antiserum (1/400) and an undiluted anti-P. acnes MAb hybridoma culture supernatant (QUBPa3) before incubation with a secondary antibody conjugated to a fluorescent dye (FITC). Note cocci and coryneform morphology of Staphylococcus spp. and $\mathrm{P}$. acnes respectively, which allows visual differentiation of the organisms.

as intramedullary nails and devices used in other areas of medicine, such as prosthetic heart valves.

\section{DISCUSSION}

Although IFM seems to be the most appropriate nonculture method currently available for the reliable detection of prosthetic hip biofilms, it is important to recognize that continuing technological advances likely are to develop improved or alternate nonculture approaches to those used currently. In particular, real-time PCR offers a notable improvement on conventional PCR methods because of its capacity for greatly reduced assay times and postamplification analysis. ${ }^{17}$ This particularly could be advantageous for the detection of prosthetic hip infections. The method also offers increased specificity and sensitivity, as well as the capacity to identify background amplification products based on quantification and melting curve analysis. Currently, the main limitation with this approach is the relatively expensive nature of the technology, which prevents it from being a routine feature for many diagnostic laboratories. Other developments in the molecular field that are likely to directly impact the detection of prosthetic hip 
infections include the use of DNA microarray or DNA chip technology. ${ }^{3,44}$ For example, the use of diagnostic microarrays containing species-specific $16 \mathrm{~S}$ rDNA oligonucleotide sequences (20-25 nucleotides) from a wide range of pathogens would allow the simultaneous detection and identification of organisms in one experiment. This greatly would reduce the time taken for diagnosis compared to conventional culture-based methods. Currently, the use of DNA-microarray technology is not financially viable for most diagnostic laboratories. Furthermore, for an 'average' laboratory, setting up an 'in-house' microarray does require a great level of expertise ranging from the construction of an arraying robot to the design of multiple species-specific 16S rDNA oligonucleotides and computer-based analysis and interpretation of the output data. ${ }^{35}$ Despite these current economic and practical limitations, microarrays likely are to become routine diagnostic tools within every laboratory in the future as methodologies improve and equipment costs decrease.

In the more immediate future, now that we have established and validated the IFM-based approach for the detection of prosthetic hip biofilms, we hope to streamline the technique so that the time from receipt of the retrieved prosthesis to identification of infection can be considerably reduced. Currently, we are optimizing our washing procedures for the glass slides and eliminating the secondary antibody incubation step by directly labeling our MAb and polyclonal antisera with FITC and TRITC, respectively. This will reduce our current processing time for the procedure (Fig 1), and also further simplify the technique. Ultimately, the goal is to develop a rapid IFM-based method to enable detection of infection before the end of surgery, guiding the clinician on the most appropriate course of treatment such as a delayed two-stage revision procedure. ${ }^{46}$

\section{References}

1. Amann R, Fuchs BM, Behrens S: The identification of microorganisms by fluorescence in situ hybridization. Curr Opin Biotechnol 12:231-236, 2001.

2. Atkins BL, Athanasou NA, Deeks JJ, et al: Prospective evaluation of criteria for microbiological diagnosis of prosthetic joint infection at revision arthroplasty. The OSIRIS collaborative study group. J Clin Microbiol 19:265-275, 1998.

3. Bodrossy L, Sessitsch A: Oligonucleotide microarrays in microbial diagnostics. Curr Opin Microbiol 7:245-254, 2004.

4. Borst A, Box ATA, Fluit AC: False-positive results and contamination in nucleic acid amplification assays: Suggestions for a prevent-and-destroy strategy. Eur J Clin Microbiol 23:288-299, 2004.

5. Branson JJ, Goldstein WM: Primary total hip arthroplasty. AORN J 78:947-943; 956-969, 2003.

6. Clarridge III JE: Impact of $16 \mathrm{~S}$ rRNA gene sequence analysis for identification of bacteria on clinical microbiology and infectious disease. Clin Microbiol Rev 17:840-862, 2004.

7. Dupont JA: Significance of operative cultures in total hip arthroplasty. Clin Orthop 211:122-127, 1986.

8. Elliott TSJ, Tebbs SE, Moss HA, et al: A novel serological test for the diagnosis of central venous catheter-associated sepsis. J Infect 40:262-266, 2000.

9. Fuchs BM, Glockner FO, Wulf J, Amann R: Unlabeled helper oligonucleotides increase the in situ accessibility to $16 \mathrm{~S}$ rRNA of fluorescently labeled oligonucleotide probes. Appl Environ Microbiol 66:3603-3607, 2000.

10. Harris KA, Hartley JC: Development of broad-range 16S rDNA PCR for use in the routine diagnostic clinical microbiology service. J Med Microbiol 52:685-691, 2003.

11. Hogan D, Kolter R: Why are bacteria refractory to antimicrobials? Curr Opin Microbiol 5:472-477, 2002.

12. Johnson JR: Development of polymerase chain reaction-based assays for bacterial gene detection. J Microbiol Methods 41:201-209, 2000.

13. Kaltas DS: Infection after total hip arthroplasty. Ann R Coll Surg Engl 86:267-271, 2004.

14. Kolbert CP, Persing DH: Ribosomal DNA sequencing as a tool for identification of bacterial pathogens. Curr Opin Microbiol 2:229305, 1999.

15. Levitsky KA, Hozack WJ, Balderston RA, et al: Evaluation of the painful prosthetic joint. Relative value of bone scan, sedimentation rate, and joint aspiration. J Arthroplasty 6:237-244, 1991.

16. MacDonald R, Brozel VS: Community analysis of bacterial biofilms in a stimulated recirculating cooling-water system by fluorescent in situ hybridization with rRNA-targeted oligonucleotide probes. Water Res 34:2439-2446, 2000.

17. Mackay IM: Real-time PCR in the microbiology laboratory. Clin Microbiol Infect 10:190-212, 2004.

18. Mahenthiralingam E, Bischof J, Byrne SK, et al: DNA-based diagnostic approaches for identification of Burkholderia cepacia complex, Burkholderia vietnamiensis, Burkholderia multivorans, Burkholderia stabilis, and Burkholderia cepacia genomovars I and III. J Clin Microbiol 38:3165-3173, 2000.

19. Markoulatos P, Siafakas N, Moncany M: Multiplex polymerase chain reaction: A practical approach. J Clin Lab Anal 16:47-51, 2002.

20. McDowell A, Valanne S, Ramage G, et al: Propionibacterium acnes types I and II represent phylogenetically distinct groups. J Clin Microbiol 43:326-334, 2005.

21. Millar BC, Moore JE: Molecular diagnostics: Current options. Methods Mol Biol 266:139-166, 2004.

22. Millar BC, Xu J, Moore JE: Risk assessment models and contamination management: Implications for broad-range ribosomal DNA PCR as a diagnostic tool in medical bacteriology. J Clin Microbiol 40:1575-1580, 2002.

23. Moter A, Göbel UB: Fluorescence in situ hybridization (FISH) for direct visualization of microorganisms. J Microbiol Methods 41:85$112,2000$.

24. Mulcahy DM, Fenelon GC, McInerney DP: Aspiration arthrography of the hip joint: Its uses and limitations in revision hip surgery. J Arthroplasty 11:64-68, 1996.

25. Norden RA: Peptide nucleic acid (PNA): Its medical and biotechnical applications and promise for the future. Fed Am Soc Exp Biol 14:1041-1060, 2000.

26. Pandey R, Berendt AR, Athanasou NA: Histological and microbiological findings in non-infected and infected revision arthroplasty tissues: The OSIRIS collaborative study group. Oxford Skeletal Infection Research and Intervention Service. Arch Orthop Trauma Surg 120:570-574, 2000.

27. Pellicci PM, Wilson Jr PD, Sledge CB, et al: Revision total hip arthroplasty. Clin Orthop Relat Res 170:34-41, 1982.

28. Perry-O'Keefe H, Rigby S, Oliveira K, et al: Identification of indicator microorganisms using a standardized PNA FISH method. J Microbiol Methods 47:281-292, 2001.

29. Rafiq M, Worthington T, Tebbs SE, et al: Serological detection of Gram-positive bacterial infection around prostheses. J Bone Joint Surg 82B:1156-1161, 2000.

30. Ramage G, Patrick S, Houston S: Combined fluorescent in situ hybridization and immunolabeling of Bacteroides fragilis. J Immunol Methods 212:139-147, 1998. 
31. Ramage G, Tunney MM, Patrick S, Gorman SP, Nixon JR: Formation of Propionibacterium acnes biofilms on orthopaedic biomaterials and their susceptibility to antimicrobials. Biomaterials 19:3221-3227, 2003.

32. Schmidt TM, Pace B, Pace NR: Detection of DNA contamination in Taq polymerase. Biotechniques 11:176-177, 1991.

33. Stirling A, Worthington T, Rafiq M, Lambert PA, Elliott TSJ: Association between sciatica and Propionibacterium acnes. Lancet 357:2024-2025, 2001.

34. Takei H, Pioletti DP, Kwon SY, Sung KL: Combined effect of titanium particles and TNF-alpha on the production of IL- 6 by osteoblast-like cells. J Biomed Mater Res 52:382-387, 2000.

35. Thompson A, Lucchini S, Hinton JCD: It's easy to build your own microarrayer. Trends Microbiol 9:154-156, 2001

36. Trampuz A, Osmon DR, Hanssen AD, Steckelberg JM, Patel R: Molecular and antibiofilm approaches to prosthetic joint infection. Clin Orthop 414:69-86, 2003.

37. Tunney MM, Patrick S, Curran MD, et al: Detection of prosthetic hip infection at revision arthroplasty by immunofluorescence microscopy and PCR amplification of the bacterial 16S rDNA gene. J Clin Microbiol 37:3281-3290, 1999.

38. Tunney MM, Patrick S, Gorman S, et al: Improved detection of infection in hip replacements: A currently underestimated problem. J Bone Joint Surg 80B:568-572, 1998

39. Virolainen P, Lahteenmaki H, Hiltunen A, et al: The reliability of diagnosis of infection during revision arthroplasties. Scand J Surg 91:178-181, 2002.

40. Wagner M, Horn M, Daims H: Fluorescence in situ hybridization for the identification and characterization of prokaryotes. Curr Opin Microbiol 6:302-309, 2003.

41. Widjojoatmodjo MN, Fluit AC, Verhoef J: Rapid identification of bacteria by PCR-single strand conformation polymorphism. J Clin Microbiol 32:3002-3007, 1994.

42. Xu J, Millar BC, Moore JE, et al: Employment of broad-range 16S rDNA PCR to detect etiological agents of infection from clinical specimens in patients with acute meningitis-rapid separation of $16 \mathrm{~S}$ rDNA PCR amplicons without the need for cloning. J Appl Microbiol 94:197-206, 2003.

43. Yang S, Rothman RE: PCR-based diagnostics for infectious diseases: Uses, limitations, and future applications in acute-care settings. Lancet Infect Dis 4:337-348, 2004.

44. Ye RW, Wang T, Bedzyk L, Crocker KM: Applications of DNA microarrays in microbial systems. J Microbiol Methods 47:257272, 2001.

45. Yugueros J, Temprano A, Sanchez M, Luengo JM, Naharro G: Identification of Staphylococcus spp. by PCR-restriction fragment length polymorphism of gap gene. J Clin Microbiol 39:3693-3695, 2001.

46. Zimmerli W, Ochsner PE: Management of infection associated with prosthetic joints. Infection 31:99-108, 2003. 